\title{
Editorial
}

\section{Dental Implant Complication Database}

Although the high predictability and long-term success rate of dental implants is well documented in the literature, complications and failures do occur. Success and predictability of dental implants have been well documented throughout the years with purported success rates of more than $90 \%{ }^{1}$ The amount of adverse events will occur in proportion with the increase in the volume of dental implants being placed. Two recent systematic reviews and meta-analyses reported that the prevalence of peri-implantitis was present in approximately $10 \%$ of implants and $20 \%$ of patients 8 to 10 years after implant placement. ${ }^{2}$ Hebballi et al $^{3}$ studied the dangers of dental devices as reported in the Food and Drug Administration Manufacturer and User Facility Device Experience

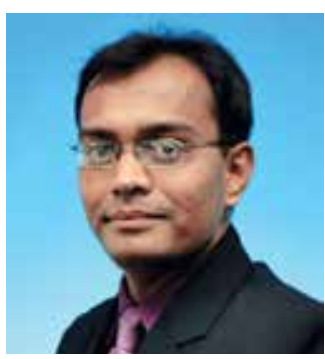
Database. The study highlighted the adverse events (AE ) of the dental devices reported in the Food and Drug Administration Manufacturer and User Facility Device Experience (MAUDE) database. The total AE reports are having continuous increasing trend from the year 1996 to 2011 in contrast to dental AE reports those are having sudden drop with decreasing trend since 1997 to 2002, followed by gradual increase in trend till 2011. Although the exact nature and quantum of dental devise complications cannot be figured-out due to varied nature of AE reporting rules and laws worldwide, the possibility of evolving the trend of dental AE reporting cannot be denied. The results indicated that, out of total dental AEs, $53.5 \%$ pertained to endosseous implants.

There are plethora of nature of complications which are identified and standardized by various authors to simplify and classify implant failures. A revised success criteria of $85 \%$ at the end of 5-year observation period and $80 \%$ at the end of 10 -year observation has been suggested. ${ }^{4}$ Clinical signs of infection with classic symptoms of inflammation, high plaque and gingival indices, pocketing, bleeding, suppuration, attachment loss, radiographic peri-implant radiolucency, presence of glaucomatous tissue upon removal. The failure may be infectious or traumatic and may be classified as biological, mechanical, iatrogenic failures or inadequate patient adaptation. According to clinical and radiographic status, the implant complications are classified as: Ailing implants, failing implants, failed implants, surviving implants. ${ }^{5}$ There is a positive learning curve in implant dentistry, represented in higher survival rates and lower complication rates reported in more recent clinical studies. However, due to rapid growth in implant-industry (including the manufacturers, funding bodies, research/teaching institutions and private clinics), there is a rapid number of increase in the trainees and newly trained practitioners, who are providing the implant treatment to the patients. 5 This will naturally lead to increase in implant failure rate due to lack of experience and expertize in the field.

In addition to MAUDE database, huge information about the dental device complications is available in the scientific literature like in scientific peer-reviewed journal articles, conference reports and titles. The nature and quantum of complications can be evaluated based on these publications. Prospective registry of the clinical trials in healthcare and mandatory good clinical practice certificate are important changing trends in healthcare research. The universal clinical database needs to be set on the single common platform to register the dental AEs worldwide which are later be updated automatically time to time. Thus, the universal norms or rules and regulations can be set to know the actual figures of dental implant-related AEs.

\section{References}

1. Adell R, Lekhom U, Rockler B, Brånemark PI. A 15-year study of osseointegrated implants in the treatment of the edentulous jaw. Int J Oral Surg 1981 Dec;10(6):387-416.

2. Mombelli A, Müller N, Cionca N. The epidemiology of peri-implantitis. Clin Oral Implants Res 2012 Oct;23(Suppl 6):67-76.

3. Hebballi NB, Ramoni R, Kalenderian E, Delattre VF, Stewart DC, Kent K, White JM, Vaderhobli R, Walji MF. The dangers of dental devices as reported in the Food and Drug Administration Manufacturer and User Facility Device Experience Database. J Am Dent Assoc 2015 Dec;146(2):102-110.

4. Smith DE, Zarb GA. Criteria for success of osseointegrated endosseous implants. J Prosthet Dent 1989 Nov;62(5):567-572.

5. Patil PG. Dental implant complications and need of publishing. Int J Prosthodont Restor Dent 2014;4(1):V.

Pravinkumar G Patil

Managing Editor

International Journal of Prosthodontics and Restorative Dentistry School of Dentistry, International Medical University

Kuala Lumpur, Malaysia 